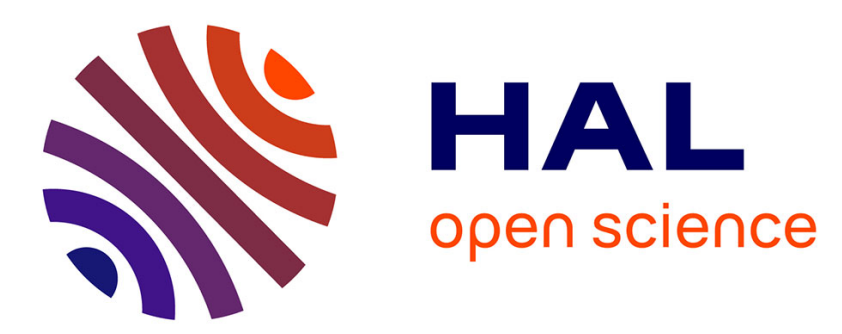

\title{
Formulation de déformation représentative et de vitesse de déformation représentative par indentation sphérique
} Liva Rabemananjara, Gerard Mauvoisin, Xavier Hernot, Adinel Gavrus, Jean Marc Collin

\section{- To cite this version:}

Liva Rabemananjara, Gerard Mauvoisin, Xavier Hernot, Adinel Gavrus, Jean Marc Collin. Formulation de déformation représentative et de vitesse de déformation représentative par indentation sphérique. Matériaux \& Techniques, 2015, 103 (6), pp.602. 10.1051/mattech/2015039 . hal01302343

HAL Id: hal-01302343

https://hal-univ-rennes1.archives-ouvertes.fr/hal-01302343

Submitted on 22 Jul 2016

HAL is a multi-disciplinary open access archive for the deposit and dissemination of scientific research documents, whether they are published or not. The documents may come from teaching and research institutions in France or abroad, or from public or private research centers.
L'archive ouverte pluridisciplinaire HAL, est destinée au dépôt et à la diffusion de documents scientifiques de niveau recherche, publiés ou non, émanant des établissements d'enseignement et de recherche français ou étrangers, des laboratoires publics ou privés. 


\title{
Formulation de déformation représentative et de vitesse de déformation représentative par indentation sphérique
}

\author{
Liva Rabemananjara $^{1}$, Gérard Mauvoisin ${ }^{1,2}$, Xavier Hernot ${ }^{1,2}$, Adinel Gavrus ${ }^{3}$ \\ et Jean Marc Collin ${ }^{1,4}$
}

Reçu le 8 juillet 2015, accepté le 24 juillet 2015

\begin{abstract}
Résumé - La déformation représentative est une des notions utilisées pour exploiter les résultats d'un essai d'indentation instrumentée. Dans ce travail, nous avons effectué une analyse numérique pour étendre le concept de déformation représentative à une vitesse de déformation représentative en indentation sphérique pour un matériau pseudo-numérique élasto-viscoplastique. Il est montré que la vitesse de déformation représentative, contrairement à celle obtenue par indentation conique, n'est plus proportionnelle à $\frac{\dot{h}}{h}$. De plus, l'utilisation de 2 courbes d'indentation à 2 vitesses de pénétration dans la procédure d'identification par analyse inverse est suffisante pour trouver la solution exacte dans le cas d'un matériau pseudonumérique défini par une loi de type Hollomon.
\end{abstract}

Mots clés : Indentation sphérique / déformation représentative / vitesse de déformation représentative / identification inverse

\begin{abstract}
Formulation of representative strain and representative strain rate in spherical indentation. One concept among many others used to exploit instrumented indentation test is the representative strain. In this work, numerical analysis was performed in order to extend the concept of representative strain to a representative strain rate for an elastic-visco-plastic material. It is shown that the proportionality with the ratio $\frac{\dot{h}}{h}$ is no longer verified, in contrast to conical indentation. Moreover, using inverse analysis with two indentation curves defined by two penetration rates is accurately sufficient to determine properly the reference parameters in the case of a pseudo-numerical material.
\end{abstract}

Key words: Spherical indentation / representative strain / representative strain rate / inverse analysis

\section{Introduction}

L'indentation instrumentée consiste à enfoncer un pénétrateur à la surface d'un matériau et à mesurer continuellement l'effort appliqué en fonction de l'enfoncement. Une des grandes problématiques de cet essai est la détermination des propriétés intrinsèques du matériau permettant de construire la courbe de la contrainte - déformation à partir des grandeurs mesurables expérimentalement. La notion de déformation représentative figure parmi les approches utilisées pour dépouiller cet essai, elle a été initiée par Tabor [1] qui a remarqué que la pression moyenne d'indentation $p_{m}$ varie linéairement en fonction de la contrainte d'écoulement du matériau indenté par un coefficient constant. Cette pression moyenne étant définie par le rapport entre l'effort et l'aire de contact projetée, qui est définie en fonction du rayon de contact réel entre l'indenteur et le matériau. Or la détermination de ce rayon de contact n'est pas évidente et demeure encore un sujet de recherche et de discussion. Pour cette raison, nous considérons dans cette étude la déformation représentative définie à partir de la courbure $C_{L}$ de la courbe d'indentation. En se basant sur la proportionnalité entre la courbure $C_{L}$ et la contrainte d'écoulement, on peut obtenir une valeur de la déformation représentative, différente de celle

\footnotetext{
${ }^{1}$ LGCGM, Université de Rennes 1, 20 avenue des buttes des Coësmes, 35708 Rennes Cedex 7, France gerard.mauvoisin@univ-rennes1.fr

2 IUT, Université de Rennes 1, 3 rue de Clos Courtel, 35700 Rennes Cedex, France

3 LGCGM, INSA de Rennes, 20 avenue des buttes des Coësmes, 35708 Rennes Cedex 7, France

4 IUT de Nantes, 2 avenue du professeur Jean Rouxel, 44475 Carquefou Cedex, France
} 
obtenue à partir de la pression de contact, déjà utilisée pour déterminer la courbe d'écrouissage des matériaux à comportement dit élasto-plastique isotrope [2-4]. L'idée peut être étendue sur des matériaux présentant une sensibilité à la vitesse de déformation en utilisant le terme de vitesse de déformation représentative. Celle-ci a fait l'objet de plusieurs études ces dernières années [5-7], les différentes formulations de la vitesse de déformation représentative proposées sont basées sur la dureté et se limitent au cas de l'indentation conique. Récemment une étude a été réalisée sur des matériaux numériques rigides viscoplastiques [8] qui a permis de proposer une nouvelle manière de calculer la déformation représentative et la vitesse de déformation représentative par indentation conique. Il a été montré que la déformation représentative et la vitesse de déformation représentative dépendent des paramètres de la loi de comportement du matériau. Ainsi, la détermination d'une déformation représentative unique est impossible avec un seul angle de cône.

Dans ce travail, l'objectif est de prendre en compte l'effet de la déformation élastique sur les déformations représentatives et les vitesses de déformation représentative calculées à partir de la courbure $C_{L}$ afin d'étendre le concept de déformation et de vitesse de déformation représentatives établi pour des matériaux rigides-viscoplastiques. L'étude est ici réalisée dans le cas de l'indentation sphérique. Le fait d'étudier le cas d'un matériau élasto-viscoplastique permet d'approcher d'avantage du comportement mécanique réel des matériaux même si dans une première approche nous nous limiterons à une loi de comportement de type Hollomon.

\section{Analyse numérique}

\subsection{Matériau d'étude}

Dans la suite, l'étude est effectuée sur un modèle numérique pour des raisons de simplicité. Nous considérons un matériau homogène et incompressible dont la loi de comportement, dépendant de la vitesse de déformation, est définie par les Équations (1a) et (1b).

$$
\begin{aligned}
d \varepsilon & =\mathrm{d} \varepsilon_{e}+\mathrm{d} \varepsilon_{P} \\
\dot{\varepsilon}_{P} & =\left(\frac{\sigma}{K \varepsilon_{P}^{n}}\right)^{\frac{1}{m}}
\end{aligned}
$$

Où $K$ est la consistance exprimée en $\mathrm{MPa} . \mathrm{s}^{\mathrm{m}}, n$ le coefficient d'écrouissage et $m$ le coefficient de sensibilité à la vitesse de déformation. La partie élastique est supposée linéaire et décrite par la loi de Hooke avec un module d'élasticité $E=2200 \mathrm{MPa}$. Ce modèle nous permet de modéliser de manière simple le comportement d'un matériau sensible à la vitesse. Pour l'étude, nous considérons un matériau de référence dont le jeu de paramètres est le suivant : $K=150 \mathrm{MPa} . \mathrm{s}^{\mathrm{m}}, n=0,2$ et $m=0,1$.

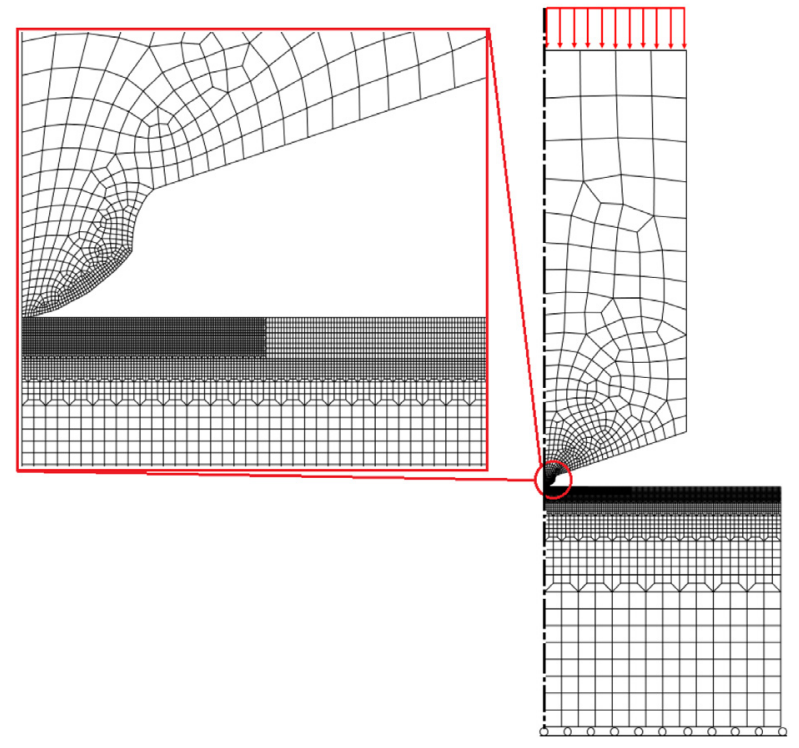

Fig. 1. Maillage utilisé pour la simulation numérique d'un essai d'indentation sphérique (Rayon de l'indenteur $r=$ $0,25 \mathrm{~mm})$.

Fig. 1. Mesh used for the numerical simulation of a spherical indentation test (indenter radius $r=0.25 \mathrm{~mm}$ ).

Des simulations d'essai d'indentation sphérique sur le matériau de référence à différentes vitesses de pénétration constantes ont été effectuées avec le code commercial ABAQUS (Fig. 1). L'indenteur est considéré élastique avec un module d'élasticité égale à $E_{\text {indenteur }}=600 \mathrm{GPa}$ et le contact entre l'indenteur et l'échantillon est modélisé par le modèle de Coulomb avec un coefficient $\mu=$ 0,1 . Le logiciel permet de calculer l'effort $F$ appliqué sur l'indenteur, le déplacement $h$ de l'indenteur et par conséquent de calculer la courbure $C_{L}$ pour chaque niveau de pénétration.

\subsection{Concept de la déformation représentative et de la vitesse de déformation représentative}

En indentation conique, il est admis depuis longtemps que les courbes d'écrouissage issues des paramètres de la loi de comportement donnant les mêmes courbes d'indentation conique se croisent en un point dont l'abscisse correspond à la déformation représentative $[1,9]$. Le raisonnement a été étendu par Moussa et al. [3] en indentation sphérique pour définir une déformation représentative moyenne. Par ailleurs, en appliquant la méthode sur un matériau dont le comportement dépend de la vitesse on pourra définir une vitesse de déformation représentative selon le même raisonnement.

\subsubsection{Domaine de solution}

Pour avoir une première idée sur ce concept, nous allons étudier la configuration du domaine défini par 

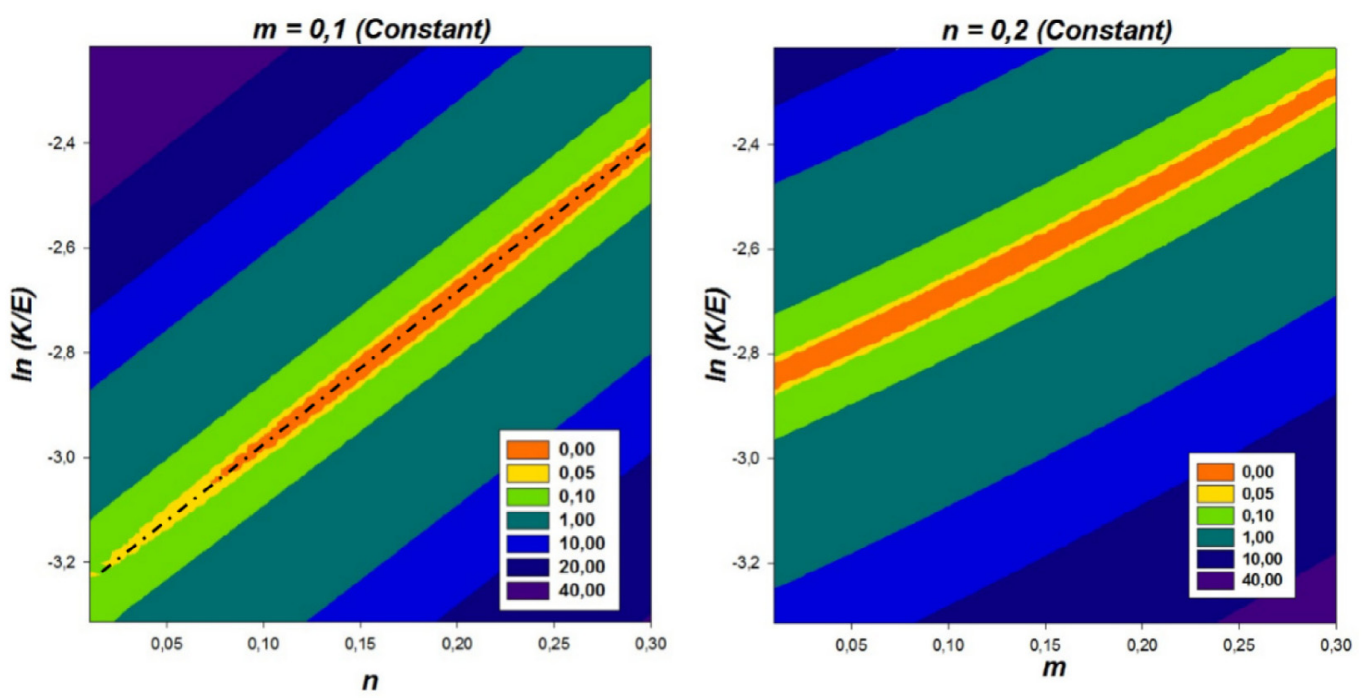

Fig. 2. Variation de la fonction objective $Q_{1}$ : (a) $m=0,1$, (b) $n=0,2$ obtenue par simulation numérique d'un essai d'indentation sphérique avec une vitesse de pénétration $h=1000 \mu \mathrm{m} . \mathrm{s}^{-1}$.

Fig. 2. Variation of the cost function $Q_{1}$ : (a) $m=0.1$, (b) $n=0.2$ obtained from numerical simulation of spherical indentation test with a penetration rate equal to $\dot{h}=1000 \mu \mathrm{m} . \mathrm{s}^{-1}$.

l'ensemble des solutions. Soit $Q_{1}$ la fonction objective qui caractérise l'écart entre l'effort de référence $\left(F^{\text {réf }}\right)$ et celui calculé $\left(F^{\mathrm{cal}}\right)$ :

$$
Q_{1}=\sqrt{\frac{1}{N} \sum_{i=1}^{N}\left(F^{\text {réf }}-F^{\text {cal }}\right)^{2}}
$$

Où $N$ représente le nombre de points expérimentaux. Dans le cas où l'on a deux vitesses de pénétration, c'est la fonction objective $Q_{2}$ qui sera considérée :

$$
\begin{aligned}
Q_{2}= & \frac{1}{2}\left\{\sqrt{\frac{1}{h_{\max }^{\mathrm{vit} 1}} \int_{0}^{h_{\max }}\left(F_{\text {ref }}^{\mathrm{vit} 1}-F_{\mathrm{cal}}^{\mathrm{vit} 1}\right)^{2} \mathrm{~d} h}\right. \\
& \left.+\sqrt{\frac{1}{h_{\max }^{\mathrm{vit} 2}} \int_{0}^{h_{\max }}\left(F_{\mathrm{ref}}^{\mathrm{vit} 2}-F_{\mathrm{cal}}^{\mathrm{vit} 2}\right)^{2} \mathrm{~d} h}\right\}
\end{aligned}
$$

La Figure 2a montre l'évolution de la fonction objective $Q_{1}$ en fonction de $K$ et $n$ pour une valeur de $m=0,1$ fixée. On constate, de manière similaire à Moussa et al. [3], qu'il existe des jeux de paramètres situés au fond de la vallée qui donnent des courbes d'indentation proches. En revanche, sur la Figure 2b, on constate qu'il existe une multitude de valeurs de $m$ qui minimisent la fonction objective $Q_{1}$. Ces figures ont été obtenues en effectuant des grilles de simulations numériques pour les plages de valeurs des paramètres de la loi de comportement indiquées par les axes des graphes.

D'après la Figure 3, le domaine de solution est réduit en utilisant deux vitesses de pénétration. Dans ce cas, l'unicité de la solution est assurée et comme nous le constatons au niveau des résultats de l'identification inverse en utilisant la méthode de simplex (Tab. 1), quels

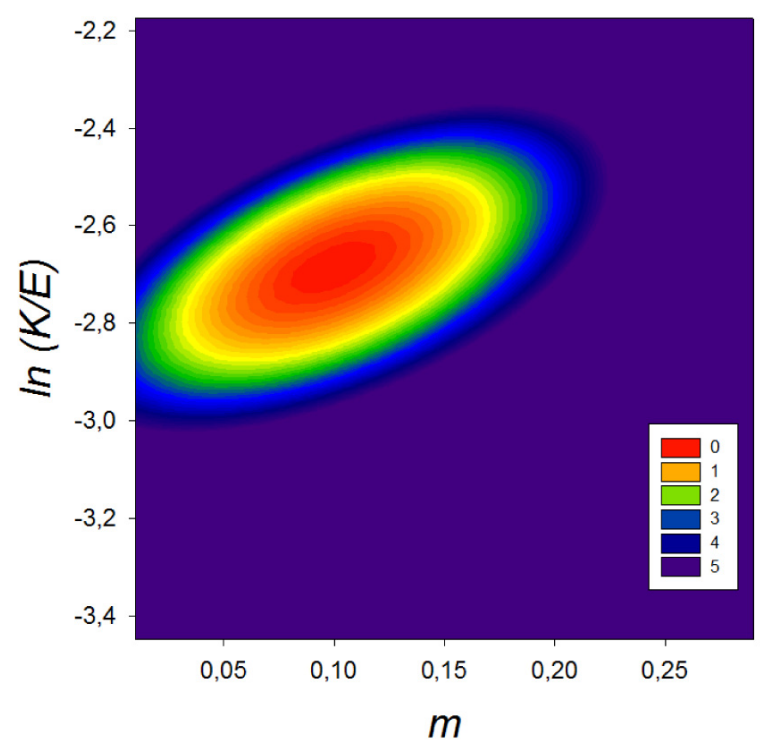

Fig. 3. Variation de la fonction objective $Q_{2}$ pour une valeur de $n$ fixé à 0,2 obtenue à partir de 2 simulations d'indentation sphérique avec des vitesses de pénétration respectivement égales à $h_{1}=10 \mu \mathrm{m} \cdot \mathrm{s}^{-1}$ et $h_{2}=1000 \mu \mathrm{m} \cdot \mathrm{s}^{-1}$.

Fig. 3. Variation of the cost function $Q_{2}$, corresponding to $n=$ 0.2 , obtained from numerical simulation of a spherical indentation test by considering 2 penetration rates $h_{1}=10 \mu \mathrm{m} . \mathrm{s}^{-1}$ and $h_{2}=1000 \mu \mathrm{m} . \mathrm{s}^{-1}$.

que soient les jeux de paramètre de départ pris au hasard, en utilisant deux courbes d'indentation sphérique caractérisées par 2 vitesses de pénétration, on retombe toujours sur la solution.

Dans le cas d'une identification à partir d'une courbe pseudo-expérimentale, le domaine de solution est 
Tableau 1. Résultat de l'identification par analyse inverse à partir de 2 courbes d'indentation sphérique spécifique à 2 vitesses de pénétration.

Table 1. Identification result from inverse analysis by considering two spherical indentation curves corresponding to two rates penetration.

\begin{tabular}{|c|c|c|c|}
\hline & $K\left[\mathrm{Mpa}_{\mathrm{N}} \mathrm{s}^{\mathrm{m}}\right]$ & $n$ & $m$ \\
\hline Paramètres de référence & 150,00000 & 0,20000 & 0,10000 \\
\hline \multirow{4}{*}{ Paramètres de départ pour l'identification 1} & 201,60645 & 0,20376 & 0,27142 \\
\hline & 247,45785 & 0,39509 & 0,25857 \\
\hline & 111,06843 & 0,29983 & 0,03169 \\
\hline & 240,92499 & 0,27975 & 0,01885 \\
\hline Résultat identification 1 (2 vitesses de pénétration) & 150,07909 & 0,20016 & 0,10016 \\
\hline Valeur de $Q_{2}[N]$ pour l'identification 1 & \multicolumn{3}{|c|}{$1,10574 \mathrm{E}-05$} \\
\hline \multirow{4}{*}{ Paramètres de départ pour l'identification 2} & 201,54195 & 0,31342 & 0,17809 \\
\hline & 90,69948 & 0,27482 & 0,10801 \\
\hline & 167,59968 & 0,01593 & 0,26952 \\
\hline & 237,92472 & 0,23079 & 0,16062 \\
\hline Résultat identification 2 (2 vitesses de pénétration) & 150,03218 & 0,20008 & 0,09999 \\
\hline Valeur de $Q_{2}[N]$ pour l'identification 2 & \multicolumn{3}{|c|}{$8,30929 \mathrm{E}-08$} \\
\hline
\end{tabular}

bien précis. Or, ce qui nous intéresse c'est d'identifier les paramètres de la loi de comportement à partir d'une courbe expérimentale. Dans ce cas, le domaine de solution peut s'agrandir à cause de l'incertitude des mesures expérimentales, d'où l'intérêt d'utiliser une déformation représentative [3] et une vitesse de déformation représentative.

\subsubsection{Choix d'une direction privilégiée}

Comme nous l'avons évoqué précédemment, déterminer la déformation représentative et la vitesse de déformation représentative revient alors à trouver, dans le domaine de solution, une direction où la fonction objective $Q$ varie peu. C'est-à-dire, trouver la direction d'un vecteur $\vec{T}\left(\ln \left(\frac{K}{E}\right), n, m\right)$, tel que :

$$
\vec{T}\left(\ln \left(\frac{K}{E}\right), n, m\right) \operatorname{grad}[Q]=0
$$

Selon l'idée de base sur le concept de déformation représentative qui suppose que la courbure $C_{L}$ est proportionnelle à la contrainte d'écoulement, la relation (4) est alors toujours vérifiée pour tous les paramètres appartenant au domaine de solution. Il a été montré par Dao et al. [9] que la courbure d'indentation est proportionnelle à la contrainte représentative :

$$
\frac{F}{E h^{2}} \sim \frac{\sigma_{R}}{E}
$$

Ou encore en combinant les relations (1) et (5), on a :

$$
\ln \left(\frac{F}{E h^{2}}\right) \sim n \ln \varepsilon_{P}+m \ln \dot{\varepsilon}_{P}+\ln \left(\frac{K}{E}\right)
$$

Il est essentiel de noter que d'après la relation (5), si la déformation représentative et la vitesse de déformation représentative sont constantes alors le domaine de solution sera une droite dans l'espace $\left(n, \ln \left(\frac{K}{E}\right)\right)$ pour une valeur de $m$ fixe. Le contraire a été montré pour le cas d'un matériau rigide viscoplastique [8].

Si $T_{1}, T_{2}$ et $T_{3}$ sont les coordonnées du vecteur $\vec{T}$ on pourra alors d'après la relation (3) écrire que :

$$
\begin{aligned}
T_{1}\left[\frac{\partial}{\partial n} \ln \left(\frac{F}{E h^{2}}\right)\right]+ & T_{2}\left[\frac{\partial}{\partial m} \ln \left(\frac{F}{E h^{2}}\right)\right] \\
& +T_{3}\left[\frac{\partial}{\partial \ln \left(\frac{K}{E}\right)} \ln \left(\frac{F}{E h^{2}}\right)\right]=0
\end{aligned}
$$

Finalement, pour une valeur de $m=m_{0}$ fixée, la déformation représentative plastique sera donnée par :

$$
\varepsilon_{R p}\left(m=m_{0}\right)=\exp \left\{\frac{\frac{\partial}{\partial n} \ln \left(\frac{F}{E h^{2}}\right)}{\frac{\partial}{\partial \ln \left(\frac{K}{E}\right)} \ln \left(\frac{F}{E h^{2}}\right)}\right\}
$$

En appliquant la même démarche pour une valeur de $n=$ $n$ fixée, la vitesse de déformation représentative plastique est donnée par :

$$
\dot{\varepsilon}_{R p}\left(n=n_{0}\right)=\exp \left\{\frac{\frac{\partial}{\partial m} \ln \left(\frac{F}{E h^{2}}\right)}{\frac{\partial}{\partial \ln \left(\frac{K}{E}\right)} \ln \left(\frac{F}{E h^{2}}\right)}\right\}
$$

Les relations (8) et (9) montrent que la déformation représentative et la vitesse de déformation représentative dépendent des paramètres du matériau.

\section{Résultats et discussion}

Dans ce qui suit, une identification par analyse inverse à partir d'une seule courbe d'indentation (une seule vitesse de pénétration $\dot{h}=1000 \mu \mathrm{m} . \mathrm{s}^{-1}$ ) a été réalisée dans 
Tableau 2. Résultat de l'identification par analyse inverse à partir d'une seule courbe d'indentation.

Table 2. Identification result from inverse analysis using only one indentation curve.

\begin{tabular}{|c|c|c|c|}
\hline & $K\left[\mathrm{Mpa} . \mathrm{s}^{\mathrm{m}}\right]$ & $n$ & $m$ \\
\hline Paramètres de référence & 150,00000 & 0,20000 & 0,10000 \\
\hline \multirow{4}{*}{ Paramètres de départ pour l'identification } & 192,00000 & 0,09000 & 0,08792 \\
\hline & 120,00000 & 0,01000 & 0,19387 \\
\hline & 234,00000 & 0,38000 & 0,18857 \\
\hline & 229,00000 & 0,14000 & 0,08546 \\
\hline Résultat identification (1 vitesse de pénétration) & 154,00000 & 0,22000 & 0,19884 \\
\hline Valeur de $Q_{1}[\mathrm{~N}]$ & \multicolumn{3}{|c|}{$1,53944 \mathrm{E}-03$} \\
\hline
\end{tabular}

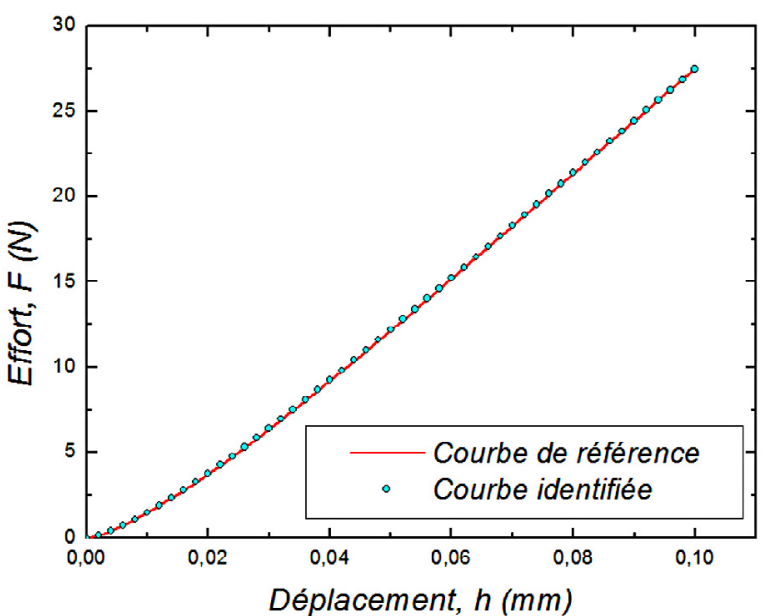

Fig. 4. Comparaison entre la courbe d'indentation de référence et la courbe obtenue par simulation numérique à partir du jeu de paramètre identifié présenté dans le Tableau 2.

Fig. 4. Comparison between the reference indentation curve and identified curve corresponding to the identified parameters given in Table 2.

le but de trouver un autre jeu de paramètres, différent de la référence mais qui donne des courbes d'indentation proches (Fig. 4). Le résultat du calcul inverse est récapitulé dans le Tableau 2.

Dans les Figures 5a et 5b sont tracées les valeurs de la déformation représentative et de la vitesse de déformation représentative calculée à partir des paramètres de référence et les paramètres identifiés du le Tableau 2. La Figure 5a montre que la déformation représentative varie en fonction de la pénétration pour un matériau dont le comportement dépend de la vitesse de déformation, ce qui a aussi été le cas pour un matériau élasto-plastique [3]. D'après la Figure 2a, la déformation représentative qui est définie par la pente du fond de la vallée est constante, or comme nous le voyons dans la Figure 5a, la déformation représentative n'est pas la même pour les paramètres de référence et les paramètres identifiés. La seule explication possible à cette contradiction est que la déformation représentative dépend aussi de la valeur de $m$; autrement dit, l'ellipse qui définit l'ensemble des solutions tourne en fonction de la valeur de $m$. Concer- nant la vitesse de déformation représentative, l'existence de deux valeurs calculées est évidente et s'explique par le fait que non seulement le fond de la vallée est plat mais il est aussi incurvé. De plus, la proportionnalité avec le rapport $\frac{\dot{h}}{h}$ n'est plus valide, ce qui est peut-être la raison pour laquelle il existe peu de travaux dédiés à la formulation de la vitesse de déformation représentative en indentation sphérique.

Cette complexité de l'indentation sphérique peut être vue cependant comme un avantage puisque la différence de la déformation représentative avec celle de la référence est due à la valeur de $m$. Il est alors possible à partir de cette différence (différence entre la déformation représentative calculée à partir de la référence et celle calculée à partir des paramètres identifiés) de corriger la valeur de $m$. Si l'on connaît bien sûr la valeur réelle de la déformation représentative du matériau.

\section{Conclusion}

Dans ce travail, le concept de déformation représentative initialement prévu pour les matériaux élasto-plastique a été étendu pour une vitesse de déformation représentative par indentation sphérique. Il a été vu numériquement que l'essai d'indentation sphérique est un essai riche en information et que l'utilisation de deux vitesses de pénétration permet certainement d'identifier par analyse inverse les jeux de paramètres d'une loi de comportement de type Hollomon. Un calcul de la déformation et de la vitesse de déformation par la méthode du gradient a été proposé à partir des jeux de paramètres qui donnent les mêmes courbes d'indentation, notamment les jeux de paramètres de référence et un jeu de paramètres identifiés par analyse inverse à partir d'une seule courbe d'indentation (une seule vitesse de pénétration). Il a été montré que la déformation représentative calculée dépend de la valeur de $m$, soulignant ainsi la complexité de l'indentation sphérique. De plus, il a été montré que la vitesse de déformation représentative par indentation sphérique n'est plus proportionnelle à $\frac{\dot{h}}{h}$. 

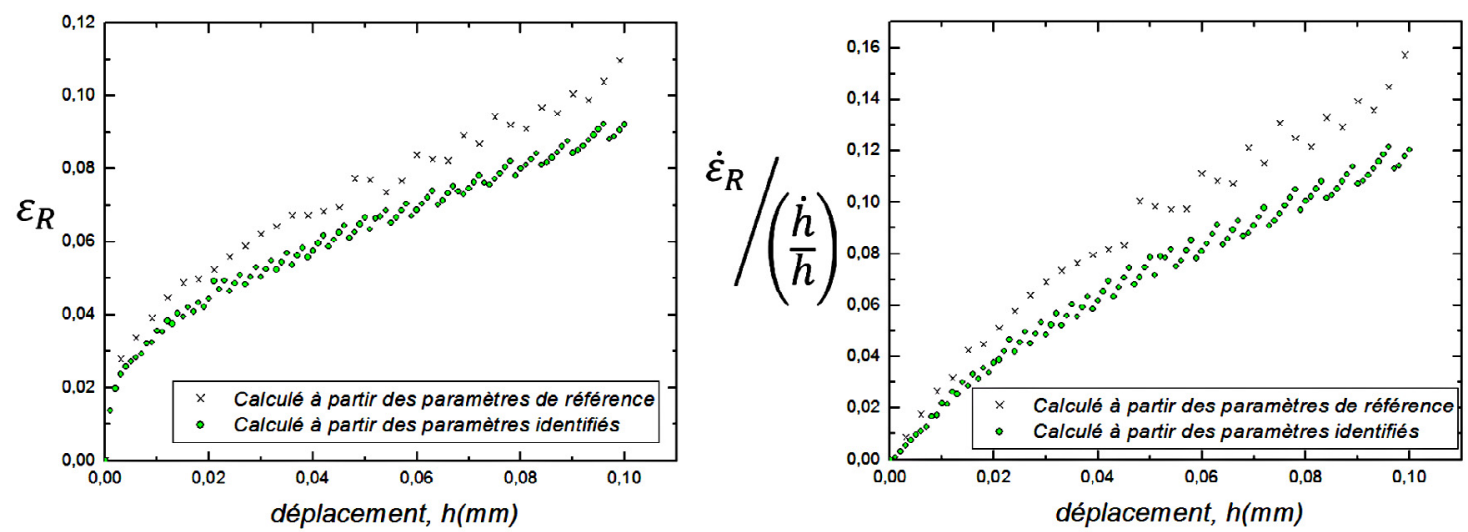

Fig. 5. (a) Déformation représentative et (b) vitesse de déformation représentative en fonction de la profondeur d'indentation obtenue à partir des jeux de paramètre de référence et les paramètres identifiés donnés dans le Tableau 1.

Fig. 5. (a) Representative strain and (b) representative strain rate function of indentation penetration obtained from the reference set of parameters and the identified parameters given in Table 1.

\section{Références}

[1] A. Atkins, D. Tabor, J. Mech. Phys. Solids 13 (1965) 149-164

[2] Y. Cao, X. Qian, N. Huber, Mater. Sci. Eng. A 454 (2007) $1-13$

[3] C. Moussa, X. Hernot, O. Bartier, G. Delattre, G. Mauvoisin, J. Mater. Sci. 49 (2014) 592-603

[4] N. Ogasawara, N. Chiba, X. Chen, J. Mater. Res. 20 (2005) 2225-2234
[5] A. Elmustafa, S. Kose, D. Stone, J. Mater. Res. 22 (2007) 926-936

[6] G. Kermouche, J.L. Loubet, J.M. Bergheau, Mech. Mater. 39 (2007) 24-38

[7] G. Kermouche, J.L. Loubet, J.M. Bergheau, Mech. Mater. 40 (2008) 271-283

[8] L. Rabemananjara, X. Hernot, G. Mauvoisin, A. Gavrus, J.M. Collin, Mater. Design 68 (2015) 207-214

[9] M. Dao, N. Cholacoop, K.J. Van Vliet, T.A. Venkatesh, S. Suresh, Acta Mater. 49 (2001) 3899-3918 